\title{
Factors of Resilience in Informal Caregivers of People with Dementia from Integrative International Data Analysis
}

\author{
Karlijn J. Joling Gill Windle Rose-Marie Dröes Franka Meiland \\ Hein P.J. van Hout Janet MacNeil Vroomen Peter M. van de Ven \\ Esmé Moniz-Cook Bob Woods
}

Bangor University, Bangor, UK; VU University Medical Centre, Amsterdam, The Netherlands

\section{Key Words}

Informal caregiving $\cdot$ Resilience $\cdot$ Dementia $\cdot$ Adversity $\cdot$ Data harmonization

\begin{abstract}
Background/Aims: Although caring for a person with dementia can be stressful, some caregivers appear to experience few negative consequences to their well-being. This study aimed to examine what proportion of caregivers demonstrates resilience under different challenging circumstances and to identify factors related to their resilience. Methods: Baseline data from 4 studies from the Netherlands and UK among informal caregivers of people with dementia were harmonized and integrated. Caregiver resilience was defined as high levels of psychological well-being despite different types of high caregiving demands. Multivariate regression analyses identified factors significantly related to caregiver resilience. Results: The integrated data set included 15 harmonized variables with data from 1,048 caregivers facing a high care demand. The prevalence of resilience varied between 35 and $43 \%$, depending on the demand for high care. Being a male caregiver, caring for a female, living apart from your relative, and low caregiver burden were positively related to caregiver resilience. Conclusion: Caregivers have the capacity to demonstrate resilience despite significant challenges. This study demonstrates how harmonization of data from multiple existing studies can be used to increase power and explore the consistency of findings. This contributes to a better understanding of which factors are likely to facilitate caregiver resilience and offers insights for developing services.


Joling et al.: Factors of Resilience in Informal Caregivers of People with Dementia from Integrative International Data Analysis

\section{Introduction}

Dementia is recognized globally as a major public health priority. Across the world, the majority of the care for persons with dementia (PwD) is provided by family members [1]. These caregivers clearly play a socially important and economically valuable role within society, but the challenge of informal care provision also presents a considerable risk for negative psychosocial consequences to the caregiver, often associated with the chronic stress involved with caregiving. Compared to non-caregivers, caregivers have an increased risk of depressive symptoms and physical health problems [2,3].

Despite this, there are groups of caregivers who, even in the face of considerable caregiving demands, appear to manage relatively well [4]. This can potentially be interpreted as a sign of resilience. Little is known about resilient caregivers, who have fulfilled their care task relatively successfully, with few negative consequences for themselves. Understanding how some caregivers are able to function well and remain resilient when caring is an important step in rebalancing the current focus on stress and poor outcomes. This new approach to rethinking dementia caregiving could contribute to developing new programmes of services for those supporting PwD.

\section{Resilience in the Context of Dementia Caregiving}

Resilience is generally understood as a positive outcome despite exposure to an adversity or risk [5], and focuses on strengths rather than weaknesses. Inspired by ecological systems theory [6], Windle and Bennett [7] developed a theoretical resilience framework for caregivers. This recognizes that caregivers will draw on individual resources, but also interact with their environment by drawing on community and societal resources which may facilitate or hinder resilience. The absence of resources may lead to poor outcomes or further caring challenges. Considering this framework, resilience can be described as 'the process of negotiating, managing and adapting to significant sources of stress or trauma. Assets and resources within the individual, their life and environment facilitate this capacity for adaptation and "bouncing back" in the face of adversity' [8, p. 163]. Whilst there is currently no standardized measure of caregiver resilience, this definition might facilitate outcome measurement, since it outlines the key elements of resilience: the encounter with adversity, the ability to resist and adapt to the adversity (through a range of assets and resources), and a positive outcome in the face of such challenges.

The few studies that attempt to understand resilience in this context provide an indication of how resilience could be measured and which factors are likely to influence resilience. Recently, Cherry et al. [9] synthesized the literature to identify factors and resources associated with good outcomes in the context of dementia care and concluded that carers' resilience is supported by social and cultural factors, properties of the caring relationship, and psychological characteristics. Applying the caregiver resilience framework, a qualitative study identified from interviews with spousal dementia caregivers that staying positive, using downward comparisons with others, actively seeking knowledge, strong social relationships, and use of respite care facilitated their resilience [10]. Hindering factors included having a negative outlook, perceived loss of social relationships, and feeling isolated. Earlier quantitative studies which measured resilience with self-report instruments identified resilience as an important predictor of change in burden, life satisfaction, and perceived stress [11] and found that social support moderated this relationship [12]. These resilience measures mainly reflect inner psychological attributes such as self-esteem and mastery. Although these can be considered indicators of resilience, such measures may fail to capture the dynamic nature of resilience, as they do not describe some of its broader social determinants. Just one study attempted to identify resilience in dementia caregivers by examining a combination of 
Dementia

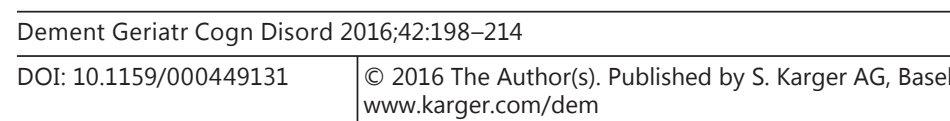

Joling et al.: Factors of Resilience in Informal Caregivers of People with Dementia from Integrative International Data Analysis

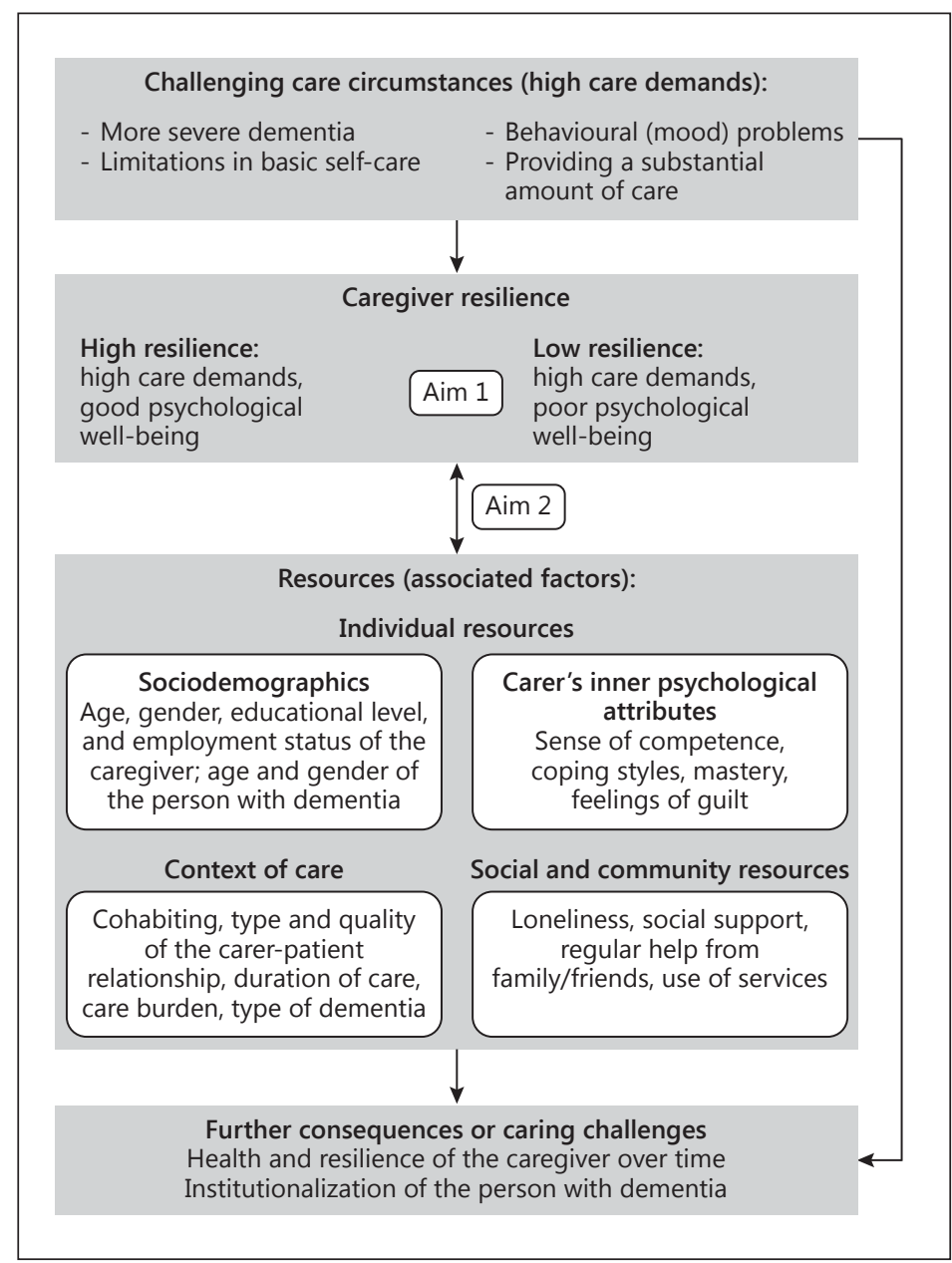

Fig. 1. Conceptual model of dementia caregiver resilience, based on the theoretical resilience framework of Windle and Bennett [7] and the research of Gaugler et al. [13]. 
Joling et al.: Factors of Resilience in Informal Caregivers of People with Dementia from Integrative International Data Analysis

internal and external resources are associated with caregiver resilience. To address these aims, this study harmonizes and integrates data from 4 studies conducted in the Netherlands and the UK.

Combining data sets from individual studies creates the opportunity to study a larger, more heterogeneous group of dementia caregivers. To be able to study resilience, a large sample size is particularly important, as it first requires the selection of the group of persons who are exposed to a significant risk or challenge. Also, combining data sets allows exploration of the consistency and generalizability of results from single studies. It responds to calls for increased data sharing and using limited resources more efficiently to answer new research questions [14]. Although the process of pooling original individual data across studies is a complicated and challenging task, it provides advantages over techniques synthesizing the relevant summary statistics, such as meta-analysis [14], and may be a way forward to advance the current state of knowledge about dementia caregiving. The present study aims to contribute to the existing literature on caregiver resilience by considering multiple samples and exploring the challenges and possibilities of harmonizing data across studies in this field. Accordingly, we first illustrate how the data were harmonized and subsequently address the two research aims.

\section{Materials and Methods}

Data Sources

Data were derived from 4 longitudinal studies conducted in the Netherlands and the UK selected as they included PwD and their primary informal caregivers living in the community at baseline and assessed key variables to measure resilience and its associated factors. The studies were the following:

- The REMCARE Study: a randomized controlled trial evaluating the (cost-)effectiveness of joint reminiscence groups for PwD and their caregivers as compared with usual care. A total of 488 dyads were recruited in the UK through NHS memory clinics and community mental health teams for older people [15].

- The FamCare Study: including a cohort of 157 informal caregivers of PwD referred to NHS community mental health teams for people with challenging behaviour in the UK (www.challengedemcare.com).

- The COMPAS Study: examined the (cost-)effectiveness of case management among community-dwelling PwD and their informal caregivers. A total of 521 dyads from regions with and without case management across the Netherlands were included [16].

- The Meeting Centres Support Programme (MCSP) studies: 2 studies evaluating the effects of the Dutch community-based MCSP compared with regular psychogeriatric day care in nursing homes. A total of 149 dyads of PwD and their informal caregivers participating in the day care programmes were included in the evaluation studies $[17,18]$.

Study Sample

For the current study, the baseline data from the study participants included in the original samples were used. This resulted in a total of 1,315 dementia caregivers in the combined data set. As the presence of significant adversity is a condition to be able to demonstrate resilience, the caregivers who faced substantial caregiving demands were selected from this data set to address the research aims (fig. 2).

\section{Definitions of Caregiver Resilience}

Caregiver resilience was defined as relatively high reported levels of psychological well-being ('positive outcome') while being exposed to various types of stressors that represent high demands on caregivers ('adversities'): caring for someone with more severe dementia, limitations in basic self-care, behavioural problems, and providing a substantial amount of care. Consequently, the caregivers who faced the high caregiving demand of interest were coded as highly resilient if they reported good psychological well-being, and as low resilient if their well-being was poor. In this way, a resilience definition was constructed for each of the 4 high caregiving demands we selected. Since it is often a combination of stressors that places a heavy burden on caregivers, we subsequently constructed a fifth resilience definition that distinguished caregivers facing more than 1 of the high caregiving demands from caregivers with no or 1 high caregiving demand. 
Fig. 2. Flow diagram of the study sample.

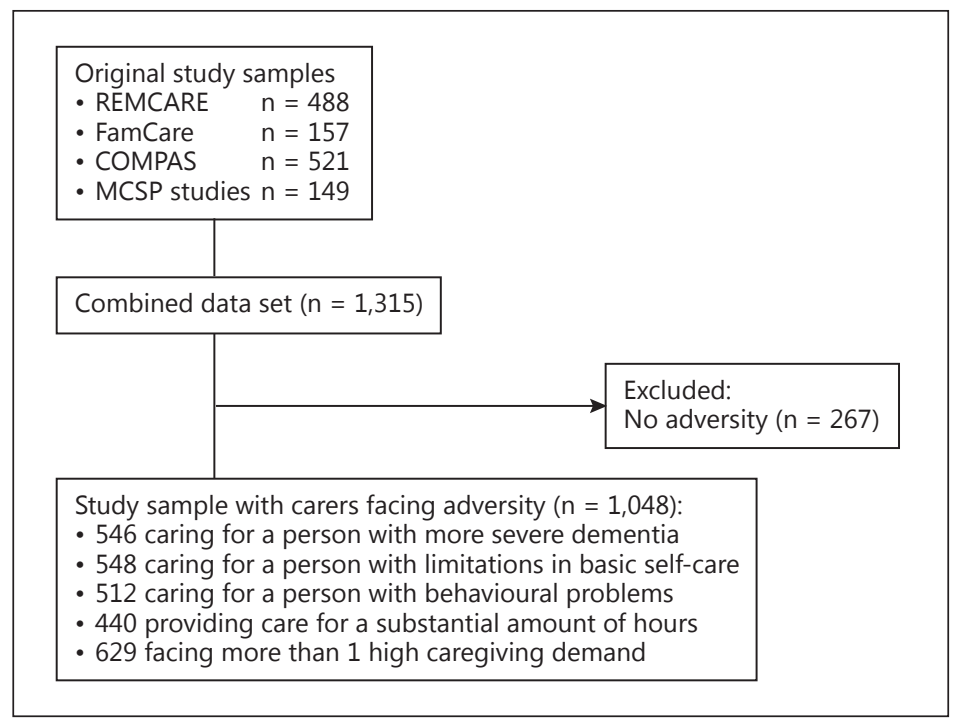

\section{Data Harmonization}

To obtain a common set of variables that could be used to assess the research aims, where feasible we followed guidelines for integrative data analysis (IDA). IDA offers methods for integrating original individual data from multiple studies for secondary analysis $[14,19]$. First, the scales and items in each of the studies were carefully reviewed. The exact wording of the relevant variables were examined by the first author and discussed with G.W. and B.W. to determine whether the variables and categories had the same face value across studies. To create the harmonized variable, categories of the original items were transformed and relabelled in each study depending on the wording and ordering. If the same item(s) was/were used, but on a different point scale, we converted scores to a common scale. Scales for care burden in each study were standardized. The harmonization process is described under Measures. Online supplementary table 1 (for all online suppl. material, see www.karger.com/doi/10.1159/000449131) gives a detailed overview of the study-specific measures together with the harmonized variables and categories.

\section{Measures}

Psychological Well-Being ('Positive Outcome')

Psychological well-being ('positive outcome') in the caregiver was measured with the General Health Questionnaire (GHQ), 12-item (FamCare and COMPAS) and 28-item (REMCARE and MCSP studies) versions [20]. The GHQ is a widely used and well-validated self-report instrument for symptoms of psychological distress. The person is asked to assess changes in his/her mood, feelings, and behaviours in the last 4 weeks. Higher scores indicate more distress. Goldberg et al. [21] compared the validity of the GHQ-12 and GHQ-28 and showed that the shorter GHQ is remarkably robust and works as well as the longer instrument to detect a case (the areas under the curve were 0.88 and 0.87 , respectively). For both versions, we used the recommended GHQ scoring method thresholds (5/6 for the GHQ-28 and 1/2 for the GHQ-12) to detect psychiatric morbidity in order to distinguish between caregivers having good and those having poor psychological well-being [21].

High Caregiving Demands ('Adversities')

More Severe Dementia. The Clinical Dementia Rating (CDR) [22], Global Deterioration Scale (GDS) [23], and Mini-Mental State Examination (MMSE) [24] were used. These measures were harmonized into a dichotomous variable with mild versus moderate or severe dementia as categories following common classifications and ranges from the literature. Perneczky et al. [25] showed that the MMSE classification has a substantial agreement with the CDR stages for the categories of mild, moderate, and severe dementia. Furthermore, Choi et al. [26] demonstrated high correlations between the GDS, CDR, and MMSE, reflecting a good concurrent validity. The mean MMSE scores in the GDS and CDR groups in this study were in line with the classification we used. 
Limitations in Basic Self-Care. We defined considerable limitations in daily functioning of PwD as having problems with performing the basic self-care tasks. In the MCSP studies, the item measuring 'help with dressing' from the Assessment Scale for Elderly Patients (ASEP) was used (no vs. some or full help needed) [27]. For the other studies, the self-care item of the EQ-5D was used (no vs. some problems or unable to wash or dress themselves) [28]. Both measures were based on caregiver proxy reports. These measures were harmonized into a dichotomous variable with no limitations versus some or severe limitations as its categories.

Behavioural/Mood Problems. To assess this high caregiving demand, we focused on the presence of significant mood symptoms in the PwD, as these were measured in all studies. REMCARE and the first MCSP study included the Cornell Scale for Depression in Dementia (CSDD) [29], and we used the cut-point of $\geq 8$, which indicates symptomatology consistent with clinically significant depression [30], to define significant mood problems in the PwD. In the FamCare and COMPAS and the second MCSP study, the Neuropsychiatric Inventory (NPI) was used to measure behavioural problems [31]. The NPI measures the frequency and severity of neuropsychiatric symptoms in 12 domains. The severity and frequency of each domain are scored and multiplied to calculate the domain composite score (range 0-12) with a score of $\geq 4$ indicating the presence of clinically relevant symptoms, and a score of $\geq 9$ indicating severe symptoms [e.g. 32, 33]. We used the NPI ratings of the combined 5 mood domains depression/dysphoria, anxiety, irritability/lability, sleep, and appetite (NPI-M) $[34,35]$ and defined the presence of significant behavioural problems as having 2 or more clinically relevant or at least 1 severe mood symptom. Both the CSDD and the NPI are valid, reliable instruments that are widely used to measure 'behavioural' disturbance in PwD.

Providing a Substantial Amount of Care. This was assessed in the REMCARE and FamCare studies by asking the caregiver how many hours he/she spent each week caring or performing care tasks for their relative. In the COMPAS study, caregivers were asked to report the hours they spent in the last week on specific tasks (household activities, personal care, transport, or visits outside the home) in which they supported the PwD or which they had taken over. The MCSP studies measured the number of days a week, and hours per day, a caregiver spent on average caring for the PwD. In each study, we converted these reports to the number of hours spent caring per week. Based on the estimate that a dementia caregiver spends around $40 \mathrm{~h}$ per week providing care duties on average, we used a threshold of $\geq 40$ care hours to identify caregivers with a substantial caring role in order to create a dichotomous variable harmonized over the studies $[36,37]$.

\section{Potential Associated Factors of Resilience}

Based on previous studies on caregiver resilience $[9,10,12,13]$ and an evidence review on resilience in later life [38], a variety of individual and external resources were considered to be potentially associated with resilience, as visualized in figure 1.

Sociodemographic Characteristics

In all studies the caregivers were asked their own and their relative's gender, age or date of birth, and employment status. The response categories for employment status differed slightly, and therefore we dichotomized this into (self-)employed or not employed to create a harmonized variable. Caregivers' educational level was assessed in the COMPAS and MCSP studies with similar categories to record the caregiver's highest completed education. We dichotomized these categories into lower or elementary versus secondary or higher education. The REMCARE and FamCare studies asked at what age the person left full time education. For these 2 studies, we used a threshold of 16 years to distinguish between caregivers with lower and higher education in the harmonized variable.

Characteristics of the Care Context

Type and Quality of the Relationship. All studies assessed the type of relationship between the caregiver and the PwD and whether they were living together. From these questions we constructed the harmonized variables 'cohabiting' (yes/no) and 'relationship to the PwD' (spouse/other). To examine the quality of the caregiver-patient relationship, the single item 'I wish that my relative and I had a better relationship' of the Short Sense of Competence Questionnaire (SSCQ) was used in the COMPAS, FamCare, and MCSP studies [39]. The answer categories ranged from 1 'agree very strongly' to 5 'strongly disagree', but in the MCSP studies the neutral option was not used. To obtain equivalent scores, we converted the item scores in the other 3 studies to a 4-point score. In the REMCARE study, the Quality of the Patient-Caregiver Relationship questionnaire (QCPR) was available [40]. We selected the QCPR item 'There is a big distance in the relationship 
Joling et al.: Factors of Resilience in Informal Caregivers of People with Dementia from Integrative International Data Analysis

between my relative and myself', which we assumed to be the most similar in meaning to the SSCQ item. The item was measured on a 5-point Likert scale and converted to a 4-point score to create the harmonized variable.

Care Burden. This was assessed in all studies, but different scales were used. In the FamCare and COMPAS studies and the second MCSP study, the NPI Caregiver Distress Scale (NPI-D) was available [31]. The NPI-D asks caregivers to rate the emotional distress they experienced in relationship to 12 neuropsychiatric symptoms on a 6-point scale (0 'not at all' to 5 'extremely distressing'). In the REMCARE study, the Relative Stress Scale (RSS) was used, a validated measure of general caregiver stress [41]. The 15 items are rated on a 5-point scale ranging from 'never' to 'always' or from 'not at all' to 'considerably'. The first MCSP study used the 13-item Caregiver Strain Index (CSI) [42]. Each item asks if a stressor or an example of a situation that depicted the stressor was present (yes/no). On all scales, higher scores indicate a higher care burden. To standardize these scales, the total scores were converted into Z-scores in each study.

Type of Dementia and Time since Symptoms. These were assessed in the COMPAS and MCSP studies. For these studies, we dichotomized dementia type into a diagnosis of Alzheimer disease versus other dementias or not specified. Time since symptoms was assessed by asking the caregiver when the first symptoms of dementia in their relative had started, and was expressed in years. Harmonization was not reached in all studies, as these 2 variables were not assessed in the REMCARE and FamCare studies.

Social and Community Resources

Use of Services. All studies asked the caregiver if their relative received home care and day care services. In the REMCARE and FamCare studies, the number of visits (home care) or days (day care) over the past 3 months was recorded, while the COMPAS study recorded the number of hours per week during this period. The MCSP studies assessed if home care services were used (yes/no) at the time of baseline assessment. All participants included in the MCSP studies were participating in some type of day care, because of the study aim. To harmonize the use of home and day care, we dichotomized the variables into yes/no. The REMCARE and FamCare studies also recorded the number of visits from a case manager in the past 3 months. In the COMPAS study this was assessed as part of the inclusion criteria. Because this service was not measured in the MCSP studies, harmonization for the primary combined analysis was not possible.

Social Support. This was measured in all studies, but with different approaches. The MCSP studies used the Social Support List (SSL-12), a valid 12-item scale assessing the level of social support by means of social interactions with members of the primary social network [43]. The other 3 studies used a single question with slightly different wording to assess whether regular help from family or friends was present (yes/no). Harmonization between these 2 approaches examining family/social support was not possible, and these variables were therefore analysed separately in an ancillary analysis.

Feelings of Loneliness. These were only measured in the COMPAS and MCSP studies, with the De JongGierveld Loneliness Scale (score 0-11), with higher scores indicating more loneliness [44]. Therefore, harmonization of this variable was not feasible across all samples.

Caregivers' Inner Psychological Attributes

Various psychological attributes of the caregiver were represented in several of the studies. Sense of competence in providing care was assessed in all studies, except REMCARE, with the SSCQ [39]. The SSCQ includes 7 items scored on a 5-point Likert scale. Because the MCSP studies used the 4-point item version, we applied the dichotomized scoring method to calculate total scores, which recodes the answer categories of 'agree very strongly/agree/neutral' into 0 and 'disagree/strongly disagree' into 1 . Higher total scores $(0-7)$ indicate a better sense of competence.

Other inner psychological characteristics of the caregivers were only available in 1 of the studies:

- $\quad$ Use of coping styles was measured in the MCSP studies with the Jalowiec Coping Scale [45].

- $\quad$ Sense of mastery was assessed in the COMPAS study with the Pearlin Mastery Scale [46].

- $\quad$ Feelings of guilt were assessed in the FamCare study with the Guilt Scale [47].

Consequently, harmonization of these characteristics in the combined data set of all samples was not possible.

\section{Statistical Analyses}

Frequencies and descriptive statistics were used to describe sample characteristics and calculate the prevalence of caregiver resilience (aim 1). To address the second aim, first, candidate variables with a significant association with resilience in univariate logistic regression $(p<0.05)$ were selected for the multivariate analysis for each of the resilience definitions. To detect multicollinearity, we checked for strong correlations 
Table 1. Description of the study samples

\begin{tabular}{|c|c|c|c|c|c|}
\hline & $\begin{array}{l}\text { REMCARE } \\
(n=379)\end{array}$ & $\begin{array}{l}\text { FamCare } \\
(\mathrm{n}=141)\end{array}$ & $\begin{array}{l}\text { COMPAS } \\
(n=405)\end{array}$ & $\begin{array}{l}\text { MCSP studies } \\
(\mathrm{n}=123)\end{array}$ & $\begin{array}{l}\text { Total } \\
(n=1,048)\end{array}$ \\
\hline \multicolumn{6}{|l|}{ Caregiver characteristics } \\
\hline Mean age (SD), years & $69.1(11.7)$ & $66.0(13.2)$ & $64.3(12.5)$ & $64.4(12.5)$ & $66.3(12.5)$ \\
\hline Female gender, $\mathrm{n}(\%)$ & $262(69.1)$ & $102(72.3)$ & $273(67.4)$ & $92(74.8)$ & $729(69.6)$ \\
\hline \multicolumn{6}{|l|}{ Education, $\mathrm{n}(\%)$} \\
\hline Elementary/lower & $167(44.1)$ & $60(42.6)$ & $61(15.1)$ & $51(41.5)$ & $339(32.3)$ \\
\hline Secondary or higher & $205(54.1)$ & $81(57.4)$ & $339(83.7)$ & $69(56.1)$ & $694(66.2)$ \\
\hline (Self-)employed, n (\%) & $63(16.6)$ & $35(24.8)$ & $160(39.5)$ & $24(19.5)$ & $282(26.9)$ \\
\hline Cohabiting, $\mathrm{n}(\%)$ & $311(82.1)$ & $102(72.3)$ & $214(52.8)$ & $86(69.9)$ & $713(68.0)$ \\
\hline Spousal relationship, n (\%) & $261(68.9)$ & $74(52.5)$ & $204(50.4)$ & $84(68.3)$ & $623(59.4)$ \\
\hline \multicolumn{6}{|l|}{ Care recipient $(P w D)$ characteristics } \\
\hline Mean age (SD), years & $77.9(7.3)$ & $80.7(7.6)$ & $80.3(7.8)$ & $75.4(7.8)$ & $78.9(7.8)$ \\
\hline Female gender, $\mathrm{n}(\%)$ & $184(48.5)$ & $84(59.6)$ & $232(57.3)$ & $57(46.3)$ & $557(53.1)$ \\
\hline Type of dementia, n (\%) & $\mathrm{n} / \mathrm{a}$ & $\mathrm{n} / \mathrm{a}$ & & & \\
\hline Alzheimer disease & & & $193(51.6)$ & $57(46.3)$ & $250(47.3)^{1}$ \\
\hline Other/not specified & & & $209(47.7)$ & $55(44.7)$ & $264(50.0)$ \\
\hline Mean years since first symptoms (SD) & $\mathrm{n} / \mathrm{a}$ & $\mathrm{n} / \mathrm{a}$ & $4.5(2.9)$ & $4.2(3.2)$ & $4.4(2.9)^{1}$ \\
\hline
\end{tabular}

$\mathrm{SD}=$ Standard deviation; PwD = person with dementia; $\mathrm{n} / \mathrm{a}=$ not available. ${ }^{1}$ Included COMPAS and MCSP study participants.

between the selected variables (Spearman's $\rho>0.7$ ). Second, the selected variables were entered in a multivariate logistic regression model and removed stepwise until all variables showed a statistically significant association with caregiver resilience $(\mathrm{p}<0.05)$. Finally, we performed 2 ancillary analyses. First, we investigated if findings were consistent across studies. Differences in prevalence rates of resilience between study samples were tested with logistic regression analyses, with study membership included in the model as an independent categorical variable. When the overall effect of study membership was significant, pairwise comparisons between studies were conducted. To determine whether differences were statistically significant, the significance level was set at $\mathrm{p}<0.0083(0.05 / 6$ comparisons for each resilience definition) after a Bonferroni correction for multiple comparisons. Next, we examined to what extent the identified factors associated with resilience in the primary analyses were consistent across studies. To do this, we examined whether the interaction between the variable and study membership was significant when included in the final multivariate model of the primary analysis. In the second analysis, we explored if the variables that could not be harmonized across all studies were of significance. For this purpose, we added the variable to the final multivariate model from the primary analysis and used the $\chi^{2}$ goodness-of-fit test to compare the 2 models in the sample(s) of caregivers for which the variable was available.

Study membership was included as a fixed factor (categorical variable) in all analyses using data from multiple studies to control for between-study heterogeneity in hypothesis testing (fixed-effect IDA [19]). Before creating the harmonized variable for severity of dementia, we used maximum likelihood imputation as implemented by the EM algorithm in SPSS to impute missing MMSE total scores in the COMPAS study. In order to replace missing MMSE scores by their most likely values while also taking into account the mechanism that generated the missingness, statistically significant predictors of the MMSE score and predictors of its missingness were included in the maximum likelihood estimation procedure to obtain the required imputed values. All analyses were performed using SPSS version 20.

\section{Results}

\section{Study Sample}

From the combined data set of 1,315 caregivers, 1,048 faced a high caregiving demand of interest and their data were used for analysis in the present study (fig. 2). The characteristics of these caregivers and their relatives with dementia are described in table 1. 
Dementia

Cognitive Disorders
Dement Geriatr Cogn Disord 2016;42:198-214

(c) 2016 The Author(s). Published by S. Karger AG, Base www.karger.com/dem

Joling et al.: Factors of Resilience in Informal Caregivers of People with Dementia from Integrative International Data Analysis

Table 2. Prevalence of high resilience, defined as good psychological well-being (GHQ score below the clinically relevant cut-point) in the face of different high caregiving demands, in the combined sample and in each study separately

\begin{tabular}{|c|c|c|c|c|c|}
\hline High resilience & REMCARE & FamCare & COMPAS & $\begin{array}{l}\text { MCSP } \\
\text { studies }\end{array}$ & $\begin{array}{l}\text { Total } \\
\text { sample }\end{array}$ \\
\hline \multicolumn{6}{|l|}{ Good psychological well-being in the face of: } \\
\hline More severe dementia in the PwD & $66(51.2)$ & $14(24.1)$ & $135(43.7)$ & $22(44.0)$ & $237(43.4)$ \\
\hline Limitations in basic self-care in the PwD & $118(52.9)$ & $19(19.2)$ & $62(41.3)$ & $32(42.1)$ & $231(42.2)$ \\
\hline Behavioural/mood problems in the PwD & $94(45.2)$ & $13(17.1)$ & $52(30.6)$ & $21(36.2)$ & $180(35.2)$ \\
\hline Providing a substantial amount of care & $106(53.0)$ & $27(31.0)$ & $22(28.2)$ & $23(30.7)$ & $178(40.5)$ \\
\hline More than 1 high caregiving demand & $121(51.3)$ & $20(20.0)$ & $74(34.4)$ & $29(37.2)$ & $244(38.8)$ \\
\hline
\end{tabular}

Figures are numbers, with percentages in parentheses. PwD = Person with dementia.

Prevalence of Resilience: Good Psychological Well-Being despite High Caregiving Demands

Table 2 shows that caregivers were most often highly resilient when caring for a person with more severe dementia (43\%), followed by caring for someone with limitations in basic self-care (42\%) and providing a high amount of care (41\%). The prevalence of resilience was lowest when dealing with behavioural/mood problems in the PwD (35\%). About39\% demonstrated high resilience when facing more than 1 high caregiving demand.

\section{Factors Associated with Caregiver Resilience}

From the list of factors potentially associated with resilience, 15 of the 24 variables were harmonized across all samples and included in the combined data set. Table 3 illustrates the factors that remained significant in the final multivariate models. A high care burden was negatively associated with high resilience for all definitions. Also, a negative association was found between cohabiting with the PwD and high resilience for all definitions, except when the high caregiving demand referred to 'providing a substantial amount of care'. Gender of the PwD (being female) or caregiver (being male) was positively associated with resilience under all challenging circumstances, except when 'caring for a person with more severe dementia'.

\section{Ancillary Analyses}

Consistency of Findings across Studies

The first ancillary analysis showed statistically significant differences between studies in the prevalence of resilience for all definitions. Online supplementary table 2 presents the results of the pairwise comparisons between studies. In summary, applying the Bonferroni correction for multiple comparisons, the prevalence of resilience was significantly higher in the REMCARE study than in the other samples for most of the resilience definitions. The FamCare sample included the lowest number of highly resilient caregivers compared to the other samples for most definitions.

Most of the variables identified to be significantly related to high resilience in the primary multivariate analyses were consistent across studies. We looked for interaction effects with study membership in the multivariate regression models and found only a significant interaction with cohabiting status for the resilience definition 'good psychological well-being in the face of more than 1 high caregiving demand' (cohabiting status $\times$ study interaction: Wald $\chi^{2}=10.16$, d.f. $=3, p=0.02$ ). Only in the COMPAS sample, cohabiting with the PwD showed a significant negative association with high resilience (adjusted OR $=0.21$, Wald $\chi^{2}=20.94$, d.f. $=1, \mathrm{p}<0.001,95 \%$ CI 0.11-0.41). 
Dementia

Cognitive Disorders

\begin{tabular}{l|l|}
\hline Dement Geriatr Cogn Disord 2016;42:198-214 \\
\hline DOI: 10.1159/000449131 & $\begin{array}{l}\text { C 2016 The Author(s). Published by S. Karger AG, Basel } \\
\text { www.karger.com/dem }\end{array}$ \\
\hline
\end{tabular}

Joling et al.: Factors of Resilience in Informal Caregivers of People with Dementia from Integrative International Data Analysis

Table 3. Results from the univariate and multivariate regression analyses

a Examining the associated factors of resilience in the combined sample

\begin{tabular}{|c|c|c|c|c|c|c|c|c|}
\hline \multicolumn{9}{|c|}{ High resilience in the face of: } \\
\hline \multicolumn{2}{|c|}{$\begin{array}{l}\text { more severe dementia } \\
(n=546)\end{array}$} & \multicolumn{4}{|c|}{$\begin{array}{l}\text { basic self-care limitations } \\
(n=548)\end{array}$} & \multicolumn{3}{|c|}{$\begin{array}{l}\text { behavioural problems } \\
(n=512)\end{array}$} \\
\hline univariate & multivariate & unis & ate & mul & riate & univ & ate & multivariate \\
\hline OR & OR & OR & $\mathrm{p}$ & OR & $\mathrm{p}$ & OR & $\mathrm{p}$ & OR \\
\hline
\end{tabular}

\section{Sociodemographics}

Age of caregiver

Male gender of caregiver

Higher education of caregiver

Caregiver (self-)employed

Age of PwD

Female gender of PwD

0.99

OR p

OR p

OR p

OR $p$

\section{Context of care}

Cohabiting

Spousal relationship

High relationship quality $(1-4)^{1}$

Higher care burden (Z-score)

$0.99 \quad 0.06$

$1.38 \quad 0.09$

$1.52<0.05^{*}$

$1.70 \quad 0.01^{*}$

$1.02 \quad 0.05$

$1.87<0.01^{*}$

$\begin{array}{ll}0.99 & 0.07\end{array}$

$1.68 \quad 0.01$

$0.99 \quad 0.06$

Community resources

Use of home care

$0.50<<0.01^{*}$

$1.630 .01^{*}$

1.64

$0.03 \quad 1.74$

$1.74 \quad 0.01^{*}$

$1.570 .03^{*}$

$1.01 \quad 0.42$

$\begin{array}{ll}1.17 & 0.45\end{array}$

$1.39 \quad 0.13$

$1.030 .03^{*}$

$2.59<0.01^{*} \quad 1.71 \quad 0.02$

Use of day care

$\begin{array}{llll}0.50 & <0.01^{*} & 0.50 & <0.01\end{array}$

$0.76 \quad 0.14$

$1.50<0.01^{*}$

$0.32<0.01^{*}$

$2.10<0.01^{*}$

(1)

$<0.01^{*}$

$0.45<0.01$

$\begin{array}{llllll}0.51 & <0.01^{*} & 0.56 & 0.01 & 0.33 & <0.01^{*} \\ 0.57 & <0.01^{*} & & & 0.52 & <0.01^{*}\end{array}$

$\begin{array}{lll}0.57<0.01 * & 1.56<0.01^{*}\end{array}$

$1.57<0.01^{*}$
$0.28<0.01^{*}$

$0.29<0.0$

$0.40<0.01^{*}$

$0.42<0.01$

b

Continued for the resilience definitions with the high caregiving demands 'substantial time spent caring' and 'more than 1 high care demand'

High resilience in the face of:
$\begin{aligned} & \text { substantial time spent caring } \\ & (\mathrm{n}=440)\end{aligned}$
$\begin{aligned} & \text { univariate } \\ & \text { OR }\end{aligned} \frac{\text { multivariate }}{\text { OR p }}$

$\begin{aligned} & \text { more than } 1 \text { high care demand } \\ & (\mathrm{n}=629)\end{aligned}$
$\begin{aligned} & \text { univariate } \\ & \text { OR p } \quad \text { multivariate }\end{aligned}$

Sociodemographics

Age of caregiv

Male gender of caregiver

Higher education of caregiver

$1.15 \quad 0.45$

$1.02 \quad 0.91$

1.010 .95

$\begin{array}{llllll}0.78 & 0.20 & 0.80 & 0.27 & 0.69 & 0.10\end{array}$

Caregiver (self-)employed

Age of PwD

Female gender of PwD

$1.01 \quad 0.5$

$\begin{array}{lrrrr}1.01 & 0.55 & 0.99 & 0.26\end{array}$

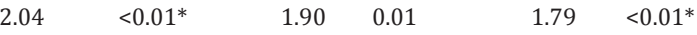

$\begin{array}{llll}1.02 & 0.93 & 1.23 & 0.27\end{array}$

$\begin{array}{llll}0.95 & 0.87 & 1.44 & 0.07\end{array}$

$\begin{array}{llll}1.01 & 0.52 & 1.02 & 0.11\end{array}$

Context of care

Cohabiting

$2.04<0.01^{*}$

$2.19<0.01^{*}$

Spousal relationship

High relationship quality $(1-4)^{1}$

Higher care burden (Z-score)

$0.60 \quad 0.1$

$\begin{array}{ll}0.60 & 0.45\end{array}$

$1.67<0.01^{*}$

$0.28<0.01$ *

.18
0.45
$0.01^{*}$
$0.01^{*}$

$0.99 \quad 0.98$

Community resources

Use of home care

$\begin{array}{ll}0.99 & 0.98 \\ 0.56 & 0.02^{*}\end{array}$

$0.51<0.01^{*}$

$0.45<0.01$

Use of day care

Resilience was measured with 5 definitions defined as good or poor psychological well-being in the face of high caregiving demands. Dependent variable: $1=$ high resilience; $0=$ low resilience. $\mathrm{OR}=0$ dds ratio; $\mathrm{PwD}=$ person with dementia. ${ }^{*} \mathrm{p}<0.05$, entered in the multivariate model. ${ }^{1}$ Higher scores are better.

Significance of Variables That Could Not Be Harmonized across all Studies

For 9 variables, harmonization was not feasible across all samples (online suppl. table 1). The second ancillary analysis examined if these variables were of significance for caregiver resilience. The $\chi^{2}$ goodness-of-fit tests indicated that a better sense of competence, greater mastery, and fewer feelings of loneliness in the caregiver were of significance for all resilience 
Table 4. Results of the $\chi^{2}$ goodness-of-fit tests

a Exploring whether the variables that could not be harmonized across all studies were of significance

\begin{tabular}{|c|c|c|c|c|c|c|c|c|c|c|c|c|}
\hline & \multicolumn{12}{|c|}{ High resilience in the face of: } \\
\hline & \multicolumn{4}{|c|}{ more severe dementia } & \multicolumn{4}{|c|}{ basic self-care limitations } & \multicolumn{4}{|c|}{ behavioural problems } \\
\hline & $\mathrm{n}$ & OR & $\chi^{2 \mathrm{a}}$ & $\mathrm{p}$ & $\mathrm{n}$ & OR & $\chi^{2 \mathrm{a}}$ & $\mathrm{p}$ & $\mathrm{n}$ & OR & $\chi^{2 \mathrm{a}}$ & $\mathrm{p}$ \\
\hline \multicolumn{13}{|l|}{ Context of care } \\
\hline Years since first symptoms ${ }^{3,4}$ & 346 & 1.01 & 0.01 & 0.91 & 213 & 0.98 & 0.11 & 0.74 & 218 & 0.97 & 0.44 & 0.51 \\
\hline Alzheimer dementia (vs. other) ${ }^{3,4}$ & 351 & 1.47 & 2.52 & 0.11 & 215 & 1.21 & 0.37 & 0.54 & 220 & 1.76 & 2.98 & 0.08 \\
\hline \multicolumn{13}{|l|}{ Inner psychological attributes } \\
\hline Sense of competence in caregiver $(0-7)^{2-4, b}$ & 408 & 1.38 & 20.80 & $<0.001$ & 322 & 1.36 & 13.75 & $<0.001$ & 302 & 1.32 & 11.17 & $<0.01$ \\
\hline Use of coping styles $(0-180)^{4, b}$ & 47 & 0.99 & 0.27 & 0.60 & 73 & 0.99 & 1.08 & 0.30 & 56 & 0.99 & 0.46 & 0.50 \\
\hline Mastery $(5-25)^{3, b}$ & 299 & 1.33 & 46.48 & $<0.001$ & 148 & 1.28 & 17.22 & $<0.001$ & 168 & 1.34 & 21.65 & $<0.001$ \\
\hline Feelings of guilt $(0-40)^{2, c}$ & 58 & 0.80 & 6.03 & 0.01 & 99 & 0.80 & 7.47 & 0.01 & 75 & 0.89 & 2.54 & 0.11 \\
\hline \multicolumn{13}{|l|}{ Social and community resources } \\
\hline Help from family/friends ${ }^{1-3}$ & 483 & 1.08 & 0.13 & 0.72 & 461 & 1.14 & 0.33 & 0.57 & 442 & 0.93 & 0.10 & 0.75 \\
\hline Social support $(12-48)^{4, b}$ & 47 & 1.14 & 6.08 & 0.01 & 73 & 1.14 & 7.14 & 0.01 & 56 & 1.05 & 0.92 & 0.34 \\
\hline Use of case management ${ }^{1-3}$ & 490 & 0.86 & 0.18 & 0.67 & 469 & 0.67 & 1.06 & 0.30 & 451 & 0.49 & 2.25 & 0.13 \\
\hline
\end{tabular}

b Continued for the resilience definitions with the high caregiving demands 'substantial time spent caring' and 'more than 1 high care demand'

\begin{tabular}{|c|c|c|c|c|c|c|c|c|}
\hline & \multicolumn{8}{|c|}{ High resilience in the face of: } \\
\hline & \multicolumn{4}{|c|}{ substantial time spent caring } & \multicolumn{4}{|c|}{ more than 1 high care demand } \\
\hline & $\mathrm{n}$ & OR & $\chi^{2 \mathrm{a}}$ & $\mathrm{p}$ & $\mathrm{n}$ & OR & $\chi^{2 \mathrm{a}}$ & $\mathrm{p}$ \\
\hline \multicolumn{9}{|l|}{ Context of care } \\
\hline Years since first symptoms 3,4 & 143 & 0.91 & 2.51 & 0.11 & 280 & 0.98 & 0.14 & 0.71 \\
\hline Alzheimer dementia (vs. other) ${ }^{3,4}$ & 144 & 1.04 & 0.01 & 0.92 & 280 & 1.46 & 1.77 & 0.18 \\
\hline \multicolumn{9}{|l|}{ Inner psychological attributes } \\
\hline Sense of competence in caregiver $(0-7)^{2-4, b}$ & 239 & 1.36 & 9.90 & $<0.01$ & 389 & 1.36 & 16.29 & $<0.001$ \\
\hline Use of coping styles $(0-180)^{4, b}$ & 71 & 0.99 & 0.16 & 0.69 & 74 & 1.00 & 0.01 & 0.92 \\
\hline Mastery $(5-25)^{3, b}$ & 77 & 1.81 & 21.71 & $<0.001$ & 212 & 1.41 & 37.93 & $<0.001$ \\
\hline Feelings of guilt $(0-40)^{2, c}$ & 87 & 0.93 & 1.16 & 0.28 & 100 & 0.88 & 3.50 & 0.06 \\
\hline \multicolumn{9}{|l|}{ Social and community resources } \\
\hline Help from family/friends ${ }^{1-3}$ & 355 & 1.04 & 0.02 & 0.88 & 537 & 1.19 & 0.66 & 0.42 \\
\hline Social support $(12-48)^{4, b}$ & 71 & 1.14 & 7.83 & 0.01 & 75 & 1.15 & 9.10 & $<0.01$ \\
\hline Loneliness of caregiver $(0-11)^{3,4, c}$ & 148 & 0.80 & 11.96 & $<0.01$ & 286 & 0.76 & 33.17 & $<0.001$ \\
\hline Use of case management $\mathrm{t}^{1-3}$ & 361 & 0.35 & 2.90 & 0.09 & 548 & 0.76 & 0.61 & 0.44 \\
\hline
\end{tabular}

The presented tests compared the model including the variables that could not be harmonized across all studies to the final multivariate model of the primary analysis. Resilience was measured with 5 definitions defined as good or poor psychological well-being in the face of high caregiving demands. Dependent variable: $1=$ high resilience; $0=$ low resilience. $\mathrm{OR}=$ Odds ratio. ${ }^{\mathrm{a}}$ Logistic regression model with 1 degree of freedom. The significant variables from the final multivariate regression model from the primary combined analysis (table 3) were included in step 1. Step 2 included the variables of interest which were not available in all samples. Models were adjusted for study membership when participants from multiple studies were included. ${ }^{\mathrm{b}}$ Higher scale scores are better. ${ }^{\mathrm{c}}$ Lower scale scores are better. ${ }^{1}$ REMCARE participants. ${ }^{2}$ FamCare participants. ${ }^{3}$ COMPAS participants. ${ }^{4}$ MCSP study participants.

definitions when adding each of these variables to the final multivariate model derived from the primary analysis and using the data of the caregivers for which the variable was available (table 4). Furthermore, fewer feelings of guilt and higher levels of social support (SSL score) were of significance in the face of some challenging circumstances. No added value was found for the type of dementia, years since first symptoms, coping styles, receiving support from a case manager, and regular help from family and friends. 
Joling et al.: Factors of Resilience in Informal Caregivers of People with Dementia from Integrative International Data Analysis

\section{Discussion}

\section{Main Findings and Interpretation}

This study contributes new insights into resilience in dementia caregiving, utilizing data from 4 studies across 2 countries. Diverse approaches to measuring resilience showed that a considerable number of caregivers are able to have good psychological well-being, despite different challenges of caring. The prevalence of resilience was comparable when high caregiving demands were present in multiple care domains. High resilience was least prevalent when behavioural/mood problems in their relative were reported and associated with gender of the caregiver and PwD as well as characteristics of the context of care (cohabiting and care burden). These insights could help to identify those at high risk and provide important orientation in priority setting for intervention by services. The exploratory ancillary analyses suggested the importance of social relationships and some inner psychological attributes of the caregiver (mastery and sense of competence). As some of these are potentially modifiable, this provides indications for practitioners to focus their support - e.g. by providing interventions aimed at improving caregivers' mastery and competence, particularly in managing challenging behaviour and (thereby) decreasing carer burden.

\section{Comparison with Other Studies}

Our estimates are quite similar to the rate of $45 \%$ reported by Gaugler et al. [13], who examined resilience, defined as lower or higher perceived burden in the face of frequent care demands, in a large sample of dementia caregivers. Several studies on resilience among older adults (not specifically caregivers) have been conducted. High scores on resilience scales have been reported and the suggestion made that these levels may remain steady or increase as older adults gain from the challenges they cope with successfully [48, 49]. Netuveli et al. [50] conducted one of the few longitudinal studies on resilience in older people, reporting a prevalence rate of $14.5 \%$. In this study, resilience was measured as a GHQ-12 score that increased after exposure to an adversity (functional limitation, bereavement or marital separation, or poverty) and returned to its pre-exposure level in the following year. Our considerably higher estimates could be due to the different types of adversity or the cross-sectional measurement of resilience in our study.

The regression analyses of the combined sample indicated the importance of cohabitation, gender, and care burden. In line with the study by Gaugler et al. [13], we found that living apart from the PwD was related to high resilience. Cohabitation was not a significant factor among caregivers providing a high amount of care, suggesting that being heavily involved rather than living together threatens caregiver resilience. Whilst Gaugler found a positive (univariate) association between being a female caregiver and resilience, our results indicated that male caregivers and persons caring for a female were more often highly resilient. This difference in findings might be due to the fact that Gaugler defined high resilience as low burden levels despite high care demands, while we used good psychological wellbeing as the positive outcome to identify highly resilient caregivers. When experiencing a stressful situation, females might be more likely to respond with depressive or anxious symptoms (captured by the GHQ). Care burden was significantly related to resilience under all challenging circumstances. Previously, Chappell and Dujela [11] found that resilience is an important predictor of change in burden. It is also known that a high caregiver burden presents a risk for negative consequences to caregivers' health [51-53]. Our study among caregivers in adversity indicates that despite relatively equal substantial care demands, the perceived burden of care varies and is a key factor for demonstrating resilience.

Prior studies among dementia caregivers provided evidence for the relevance of social supportive resources $[10,12]$. Our ancillary analysis of some of the individual studies also 
suggested that perceived low levels of social support and feelings of loneliness are related to low resilience. In contrast, this relationship was not found for the variable 'help from family and friends'. As this variable was a dichotomized measure, we hypothesize that it may not have been as sensitive as the continuous social support and loneliness measures. In addition, these analyses indicated the positive impact of inner psychological attributes such as mastery and sense of competence, and, to a lesser extent, the absence of guilt feelings. This corresponds with earlier findings from qualitative interviews reporting the importance of staying positive [10]. Previously, resilience was found to be positively related to a problem-focused coping style and the use of distancing, by not letting situations get to oneself and looking on the bright side, while escape-avoidance coping appeared to have a negative impact on resilience [54]. As males generally adopt more problem-focused coping styles and females use more emotion-focused styles when facing stress [e.g. 55], this might be one of the explanations why more males than females were highly resilient in our study. We were only able to explore the importance of coping styles in a small sample of caregivers. Possibly, this analysis might have lacked statistical power to detect a significant impact.

\section{Strengths and Limitations}

As far as we are aware, this is the first study addressing resilience simultaneously in multiple samples of dementia caregivers across nations. This enabled us to use a large, diverse sample and consider the heterogeneity of caregivers, contributing to a better understanding of the role of assets and resources related to resilience independent of specific study contexts. The use of existing data sets allowed us to add to the very limited work so far on resilience in dementia caregiving in an efficient way, but it also had its restrictions. A major challenge involved the variations across the data sets with regard to the measures that were used. Therefore, we needed to be pragmatic in measuring resilience. For example, we included only mood symptoms when examining behavioural problems in the PwD as adversity, because these symptoms were assessed in all studies. Although depression is one of the most common disturbances in PwD [e.g. 56] and puts a heavy strain on caregivers, there are also other important behavioural symptoms which are likely to increase the demand of care. Besides, the 2 scales we used might have differed in their ability to detect mood symptoms in dementia. A study which examined the effect of sertraline on depression in patients with Alzheimer disease suggested that the CSDD was more likely to detect differences in depression over time than the NPI-M [35]. This might be due to differences between items, but also because the CSDD combines patient observations with discussions with the caregiver to assess depression, whilst the NPI-M uses a caregiver interview only. It has previously been suggested that caregivers view depressed PwD as less depressed than do clinicians [57]. In our study, the prevalence of significant mood problems in the samples that used the NPI-M was indeed somewhat higher than in the samples using the CSDD - except in the FamCare sample, which showed the highest percentage of PwD with mood problems, as this study recruited people with a referral for challenging behaviour (data not shown).

We found variability between samples in resilience rates. This could be explained by differences in participants' characteristics and sampling criteria. For example, the FamCare study included caregivers of PwD who were referred for displaying challenging behaviour. FamCare caregivers were most frequently facing a high caregiving demand, and the resilience rates were lowest under all the challenging circumstances. This could indicate that this sample involved a more severely afflicted patient group compared with the other studies. Compared with the other samples, REMCARE caregivers demonstrated high resilience most frequently for all definitions. They might have experienced better psychological well-being because they cared for relatively young persons in a mild disease stage. Although we cannot be sure to what extent variations in designs and methods across studies have impacted our 
results, the use of several samples also provided the opportunity to observe these differences between caregivers. Besides, the ancillary analyses indicated that almost all factors that were identified to be significantly related to resilience in the primary analysis were consistent across studies. Only the association between cohabitation and resilience in the face of more than 1 high caregiving demand differed between studies. In the COMPAS sample, cohabiting with the PwD showed a significant negative association with high resilience, while this association was not significant in the other samples. The fact that a relatively large proportion of COMPAS caregivers were living apart from the PwD compared to the other samples might have influenced this association.

Since the studies were not originally designed to examine resilience, other factors that might be related to resilience, such as biological and physical characteristics or the presence of other major stressful life events, could have been missed. Some potential influencing factors, such as caregiving duration as well as psychological and social resources, would be useful to investigate, but they were not available for all samples and thus excluded from the primary combined analysis. The ancillary analysis using (some of) the individual samples indicated that inner psychological attributes and strong social relationships are important factors to examine in future research.

Although all study samples had longitudinal data available, we only used baseline data, as different follow-up times and the exposure to interventions during some of the studies would likely have influenced the results. Consequently, we could not demonstrate if resilience was present over time or identify which factors were key to achieve resilience. Therefore, future longitudinal research is needed to develop an understanding of how caregivers, despite significant challenges, are able to continue to function well in the long term and to determine the causal relationship to potential resources.

We tried to minimize problems of combining data sets and consider heterogeneity in studies by following IDA methods as much as possible. For example, we used fixed-effect IDA to control for between-study heterogeneity. Also, we aimed to optimally harmonize variables by examining the face validity of items, making transformations if these were highly overlapping across studies and standardizing scales if different measures were used for the same domain. This approach corresponds with previous efforts to harmonize data in a pragmatic way [58-61]. Even though limitations of our study are related to restrictions of secondary data analysis, this could also be seen as one of the strengths. Until now, research on dementia caregiving has predominantly focused on analysing data from single studies, with often relatively small, homogeneous samples. Also, existing population-based studies often do not provide enough information to study this target group thoroughly, especially around the types of caregiver challenges specifically arising from someone living with dementia. For example, in the UK, the Cognitive Function and Aging Studies (CFAS) do not ask about the type and context of care [62]. Besides, although dementia is a public health priority, the number of PwD (and their caregivers) still forms only a small percentage of the overall population. Despite its challenges, attempts to combine original data from secondary data sources may create new opportunities to test hypotheses and can provide advantages over focusing on one sample or time-consuming and expensive collection of primary data.

\section{Conclusion and Implications}

Examining this topic in multiple data sets simultaneously provides a starting point for future research. Our conceptual model reflects initial considerations of what might enable resilience for dementia caregivers, and our analyses sought to test this process. In summary, the findings indicate that although caregiving for a relative with dementia can be extremely 
Joling et al.: Factors of Resilience in Informal Caregivers of People with Dementia from Integrative International Data Analysis

challenging, a group of caregivers appear to be able to manage relatively well. Resilience was observed across samples in the face of various types of high care demands. The finding that high resilience was associated with several factors confirms the conclusion of previous studies $[10,13]$ and indicates the multidimensional nature of resilience. Future studies should investigate the potential of different services to improve caregiver resilience. A standardized resilience measure in the context of caregiving would allow more accurate measurement across populations and may facilitate tailoring of interventions. In the face of the growing numbers of people living with dementia, ensuring those who support them can remain healthy is a policy priority. There is a challenge to apply research findings to the development and implementation of interventions which could, when successfully implemented, promote resilience in caregivers and ensure that providing care for PwD remains manageable in the future.

\section{Acknowledgements}

This work was supported by the Dutch Alzheimer's Association (Alzheimer Nederland; grant No. WE.152014-05). The funder had no further role in the study design, in the collection, analysis, and interpretation of the data, in the writing of the report, and in the decision to submit the paper for publication.

\section{Disclosure Statement}

The authors declare that they have no competing interests.

\section{References}

1 World Health Organization: Dementia. A public health priority. Geneva, WHO, 2012.

-2 Joling KJ, Van Hout HP, Schellevis FG, van der Horst HE, Scheltens P, Knol DL, van Marwijk HW: Incidence of depression and anxiety in the spouses of patients with dementia: a naturalistic cohort study of recorded morbidity with a 6-year follow-up. Am J Geriatr Psychiatry 2010;18:146-153.

3 Vitaliano PP, Zhang J, Scanlan JM: Is caregiving hazardous to one's physical health? A meta-analysis. Psychol Bull 2003;129:946-972.

4 Gaugler JE, Davey A, Pearlin LI, Zarit SH: Modeling caregiver adaptation over time: the longitudinal impact of behavior problems. Psychol Aging 2000;15:437-450.

5 Masten AS: Ordinary magic. Resilience processes in development. Am Psychol 2001;56:227-238.

6 Bronfenbrenner U: Ecological models of human development; in Husen T, Postlethwaite TN (eds): International Encyclopedia of Eductation. New York, Elsevier Science, 1994, pp 1643-1647.

7 Windle G, Bennett KM: Caring relationships: how to promote resilience in challenging times; in Ungar M (ed): The Social Ecology of Resilience: A Handbook of Theory and Practice. New York, Springer, 2011, pp 219-231.

8 Windle G: What is resilience? A review and concept analysis. Rev Clin Gerontol 2011;21:152-169.

-9 Cherry MG, Salmon JM, Dickson JM, Powell D, Sikdar S, Ablett J: Factors influencing the resilience of carers of individuals with dementia. Rev Clin Gerontol 2013;23:251-266.

10 Donnellan WJ, Bennett KM, Soulsby LK: What are the factors that facilitate or hinder resilience in older spousal dementia carers? A qualitative study. Aging Ment Health 2015;19:932-939.

11 Chappell NL, Dujela C: Caregiving: predicting at-risk status. Can J Aging 2008;27:169-179.

12 Wilks SE, Croom B: Perceived stress and resilience in Alzheimer's disease caregivers: testing moderation and mediation models of social support. Aging Ment Health 2008;12:357-365.

-13 Gaugler JE, Kane RL, Newcomer R: Resilience and transitions from dementia caregiving. J Gerontol B Psychol Sci Soc Sci 2007;62:38-44.

14 Curran PJ, Hussong AM: Integrative data analysis: the simultaneous analysis of multiple data sets. Psychol Methods 2009;14:81-100.

15 Woods RT, Orrell M, Bruce E, Edwards RT, Hoare Z, Hounsome B, Keady J, Moniz-Cook E, Orgeta V, Rees J, Russell I: REMCARE: pragmatic multi-centre randomised trial of reminiscence groups for people with dementia and their family carers: effectiveness and economic analysis. PLoS One 2016;11:e0152843.

-16 MacNeil Vroomen J, Bosmans JE, van de Ven PM, Joling KJ, van Mierlo LD, Meiland FJ, Moll van Charante EP, Van Hout HP, de Rooij SE: Community-dwelling patients with dementia and their informal caregivers with and without case management: 2-year outcomes of a pragmatic trial. J Am Med Dir Assoc 2015;16:800-808. 
Joling et al.: Factors of Resilience in Informal Caregivers of People with Dementia from Integrative International Data Analysis

17 Dröes RM, Breebaart E, Meiland FJ, van Tilburg W, Mellenbergh GJ: Effect of Meeting Centres Support Program on feelings of competence of family carers and delay of institutionalization of people with dementia. Aging Ment Health 2004;8:201-211.

18 Dröes RM, Meiland FJ, Schmitz MJ, van Tilburg W: Effect of the Meeting Centres Support Program on informal carers of people with dementia: results from a multi-centre study. Aging Ment Health 2006;10:112-124.

19 Hussong AM, Curran PJ, Bauer DJ: Integrative data analysis in clinical psychology research. Annu Rev Clin Psychol 2013;9:61-89.

20 Goldberg D, Williams P: A user's guide to the General Health Questionnaire. Windsor, NFER-Nelson, 1988.

-21 Goldberg DP, Gater R, Sartorius N, Ustun TB, Piccinelli M, Gureje O, Rutter C: The validity of two versions of the GHQ in the WHO study of mental illness in general health care. Psychol Med 1997;27:191-197.

22 Morris JC: The Clinical Dementia Rating (CDR): current version and scoring rules. Neurology 1993;43:24122414.

23 Reisberg B, Ferris SH, de Leon MJ, Crook T: The Global Deterioration Scale for assessment of primary degenerative dementia. Am J Psychiatry 1982;139:1136-1139.

24 Folstein MF, Folstein SE, McHugh PR: 'Mini-mental state'. A practical method for grading the cognitive state of patients for the clinician. J Psychiatr Res 1975;12:189-198.

-25 Perneczky R, Wagenpfeil S, Komossa K, Grimmer T, Diehl J, Kurz A: Mapping scores onto stages: Mini-Mental State Examination and Clinical Dementia Rating. Am J Geriatr Psychiatry 2006;14:139-144.

-26 Choi SH, Lee BH, Kim S, Hahm DS, Jeong JH, Yoon SJ, Jeong Y, Ha CK, Nab DL: Interchanging scores between Clinical Dementia Rating scale and Global Deterioration Scale. Alzheimer Dis Assoc Disord 2003;17:98-105.

27 van der Kam P, Mol F, Wimmers MFHG: Beoordelingsschaal voor Oudere Patienten. Deventer, Van Loghum Slaterus, 1971.

28 EuroQol Group: EuroQol - a new facility for the measurement of health-related quality of life. Health Policy 1990;16:199-208.

29 Alexopoulos GS, Abrams RC, Young RC, Shamoian CA: Cornell Scale for Depression in Dementia. Biol Psychiatry 1988;23:271-284.

-30 Watson LC, Garrett JM, Sloane PD, Gruber-Baldini AL, Zimmerman S: Depression in assisted living: results from a four-state study. Am J Geriatr Psychiatry 2003;11:534-542.

31 Cummings JL, Mega M, Gray K, Rosenberg-Thompson S, Carusi DA, Gornbein J: The Neuropsychiatric Inventory: comprehensive assessment of psychopathology in dementia. Neurology 1994;44:2308-2314.

-32 Aalten P, de Vugt ME, Jaspers N, Jolles J, Verhey FR: The course of neuropsychiatric symptoms in dementia. Part II: relationships among behavioural sub-syndromes and the influence of clinical variables. Int J Geriatr Psychiatry 2005;20:531-536.

33 Selbaek G, Kirkevold Ø, Engedal K: The prevalence of psychiatric symptoms and behavioural disturbances and the use of psychotropic drugs in Norwegian nursing homes. Int J Geriatr Psychiatry 2007;22:843-849.

-34 Lyketsos CG, DelCampo L, Steinberg M, Miles Q, Steele CD, Munro C, Baker AS, Sheppard JM, Frangakis C, Brandt J, Rabins PV: Treating depression in Alzheimer disease: efficacy and safety of sertraline therapy, and the benefits of depression reduction: the DIADS. Arch Gen Psychiatry 2003;60:737-746.

35 Mayer LS, Bay RC, Politis A, Steinberg M, Steele C, Baker AS, Rabins PV, Lyketsos CG: Comparison of three rating scales as outcome measures for treatment trials of depression in Alzheimer disease: findings from DIADS. Int J Geriatr Psychiatry 2006;21:930-936.

36 Liu W, Gallagher-Thompson D: Impact of dementia caregiving: risks, strains, and growth; in Qualls WH, Zarit SH (eds): Aging Families and Caregiving. Hoboken, John Wiley \& Sons, 2009, pp 85-111.

-37 Schneider J, Hallam A, Murray J, Foley B, Atkin L, Banerjee S, Islam MK, Mann A: Formal and informal care for people with dementia: factors associated with service receipt. Aging Ment Health 2002;6:255-265.

38 Bennett KM: Emotional and personal resilience through life. Future of an ageing population: evidence review. London, Government Office for Science, 2015.

-39 Vernooij-Dassen MJ, Felling AJ, Brummelkamp E, Dauzenberg MG, van den Bos GA, Grol R: Assessment of caregiver's competence in dealing with the burden of caregiving for a dementia patient: a Short Sense of Competence Questionnaire (SSCQ) suitable for clinical practice. J Am Geriatr Soc 1999;47:256-257.

40 Spruytte N, van Audenhove C, Lammertyn F, Storms G: The quality of the caregiving relationship in informal care for older adults with dementia and chronic psychiatric patients. Psychol Psychother 2002;75:295-311.

-41 Greene JG, Smith R, Gardiner M, Timbury GC: Measuring behavioural disturbance of elderly demented patients in the community and its effects on relatives: a factor analytic study. Age Ageing 1982;11:121-126.

42 Robinson BC: Validation of a Caregiver Strain Index. J Gerontol 1983;38:344-348.

43 van Eijk LM, Kempen GI, van Sonderen FL: A short scale for measuring social support in the elderly: the SSL12-I (in Dutch). Tijdschr Gerontol Geriatr 1994;25:192-196.

44 de Jong Gierveld J, Kamphuis FH: The development of a Rasch-type Loneliness scale. Appl Psychol Meas 1985; 9:289-299.

45 Jalowiec A, Murphy SP, Powers MJ: Psychometric assessment of the Jalowiec Coping Scale. Nurs Res 1984;33: 157-161.

46 Pearlin LI, Schooler C: The structure of coping. J Health Soc Behav 1978;19:2-21.

47 Woods B: Guilt in relatives of older people in residential care. Manchester, British Congress of Gerontology, 1996.

48 Wagnild G: Resilience and successful aging. Comparison among low and high income older adults. J Gerontol Nurs 2003;29:42-49. 
Joling et al.: Factors of Resilience in Informal Caregivers of People with Dementia from Integrative International Data Analysis

49 Nygren B, Aléx L, Jonsén E, Gustafson Y, Norberg A, Lundman B: Resilience, sense of coherence, purpose in life and self-transcendence in relation to perceived physical and mental health among the oldest old. Aging Ment Health 2005;9:354-362.

-50 Netuveli G, Wiggins RD, Montgomery SM, Hildon Z, Blane D: Mental health and resilience at older ages: bouncing back after adversity in the British Household Panel Survey. J Epidemiol Community Health 2008; 62:987-991.

51 Carretero S, Garcés J, Ródenas F, Sanjosé V: The informal caregiver's burden of dependent people: theory and empirical review. Arch Gerontol Geriatr 2009;49:74-79.

52 Etters L, Goodall D, Harrison BE: Caregiver burden among dementia patient caregivers: a review of the literature. J Am Acad Nurse Pract 2008;20:423-428.

53 Schulz R, Boerner K, Shear K, Zhang S, Gitlin LN: Predictors of complicated grief among dementia caregivers: a prospective study of bereavement. Am J Geriatr Psychiatry 2006;14:650-658.

54 Garity J: Caring for a family member with Alzheimer's disease: coping with caregiver burden post-nursing home placement. J Gerontol Nurs 2006;32:39-48.

55 Garity J: Stress, learning style, resilience factors, and ways of coping in Alzheimer family caregivers. Am J Alzheimers Dis Other Demen 1997;12:171-178.

56 Lyketsos CG, Lopez O, Jones B, Fitzpatrick AL, Breitner J, DeKosky S: Prevalence of neuropsychiatric symptoms in dementia and mild cognitive impairment: results from the cardiovascular health study. JAMA 2002;288: 1475-1483.

57 Teri L, Wagner AW: Assessment of depression in patients with Alzheimer's disease: concordance among informants. Psychol Aging 1991;6:280-285.

58 Bath PA, Deeg D, Poppelaars J: The harmonisation of longitudinal data: a case study using data from cohort studies in the Netherlands and the United Kingdom. Ageing Soc 2010;30:1419-1437.

59 Minicuci N, Noale M, León Díaz EM, Gómez León M, Andreotti A, Mutafova M: Disability-free life expectancy: a cross-national comparison among Bulgarian, Italian, and Latin American older population [sic!]. J Aging Health 2011;23:629-681.

60 Pluijm SMF, Bardage C, Nikula S, Blumstein T, Jylhä M, Minicuci N, Zunzunegui MV, Pedersen NL, Deeg DJH: A harmonized measure of activities of daily living was a reliable and valid instrument for comparing disability in older people across countries. J Clin Epidemiol 2005;58:1015-1023.

61 Sikkes SAM, Visser PJ, Knol DL, de Lange-de Klerk E, Tsolaki M, Frisoni GB, Nobili F, Spiru L, Rigaud AS, Frölich L, Olde Rikkert M, Soininen H, Touchon J, Wilcock G, Boada M, Hampel H, Bullock R, Vellas B, Pijnenburg YAL, Scheltens P, Verhey FR, Uitdehaag BMJ: Do instrumental activities of daily living predict dementia at 1- and 2-year follow-up? Findings from the Development of Screening Guidelines and Diagnostic Criteria for Predementia Alzheimer's Disease study. J Am Geriatr Soc 2011;59:2273-2281.

-62 Brayne C, McCracken C, Matthews FE: Cohort profile: the Medical Research Council Cognitive Function and Ageing Study (CFAS). Int J Epidemiol 2006;35:1140-1145. 